OPEN ACCESS

Edited by:

Qiwei He,

Educational Testing Service,

United States

Reviewed by:

Xiaoou Li,

University of Minnesota Twin Cities,

United States

Chun Wang,

University of Washington

United States

*Correspondence:

Jung Yeon Park

ellie.park@kuleuven.be

Specialty section:

This article was submitted to

Quantitative Psychology and

Measurement,

a section of the journal

Frontiers in Psychology

Received: 18 November 2018 Accepted: 06 March 2019

Published: 29 March 2019

Citation:

Park JY, Cornillie F, van der Maas HLJ and Van Den Noortgate W (2019) A

Multidimensional IRT Approach for Dynamically Monitoring Ability Growth

in Computerized Practice

Environments. Front. Psychol. 10:620.

doi: 10.3389/fpsyg.2019.00620

\section{A Multidimensional IRT Approach for Dynamically Monitoring Ability Growth in Computerized Practice Environments}

\author{
Jung Yeon Park ${ }^{1 *}$, Frederik Cornillie ${ }^{1}$, Han L. J. van der Maas ${ }^{2}$ and \\ Wim Van Den Noortgate ${ }^{1}$ \\ ${ }^{1}$ Faculty of Psychology and Educational Sciences, imec-ITEC, KU Leuven, Leuven, Belgium, ${ }^{2}$ Department of Psychology, \\ University of Amsterdam, Amsterdam, Netherlands
}

Adaptive learning systems have received an increasing attention as they enable to provide personalized instructions tailored to the behaviors and needs of individual learners. In order to reach this goal, it is desired to have an assessment system, monitoring each learner's ability change in real time. The Elo Rating System (ERS), a popular scoring algorithm for paired competitions, has recently been considered as a fast and flexible method that can assess learning progress in online learning environments. However, it has been argued that a standard ERS may be problematic due to the multidimensional nature of the abilities embedded in learning materials. In order to handle this issue, we propose a system that incorporates a multidimensional item response theory model (MIRT) in the ERS. The basic idea is that instead of updating a single ability parameter from the Rasch model, our method allows a simultaneous update of multiple ability parameters based on a compensatory MIRT model, resulting in a multidimensional extension of the ERS ("M-ERS"). To evaluate the approach, three simulation studies were conducted. Results suggest that the ERS that incorrectly assumes unidimensionality has a seriously lower prediction accuracy compared to the M-ERS. Accounting for both speed and accuracy in M-ERS is shown to perform better than using accuracy data only. An application further illustrates the method using real-life data from a popular educational platform for exercising math skills.

Keywords: multidimensional IRT, Elo rating system, adaptive practice, speed-accuracy trade-off, e-learning

\section{INTRODUCTION}

Over the past decade adaptive learning systems have received an increasing attention as they enable to provide instructions tailored to the behaviors, needs, and learning pace of individual learners. In this way the learners can benefit from more personalized learning items. Therefore, it is desired for the systems to have a learner modeling method that keeps track of the learner's cognitive states and its evolution in a timely and flexible manner. In the context of computerized adaptive testing (CAT; van der Linden, 2000) the use of item response theory (IRT) is a common method to model the relationship between the learner's ability level and their responses to different measurable items.

As originally intended for high-stakes standardized tests, studies related to CAT primarily zooms in on how to increase the precision of the examinee's ability level estimate by successively rendering 
most informative items, or how to decrease the number of test items while maintaining a high level of precision in estimating the ability level. Because typically no feedback is given during the test, the true ability level is not expected to evolve. The idea of CAT also can be applied to learning environments in which learners interact with items, toward computerized adaptive practice (CAP; Klinkenberg et al., 2011). An a-priori expectation is that the learners in a learning environment, unlike in a testing environment, tend to develop their knowledge by interacting with the items rendered (and by getting feedback on their responses), and their true ability levels consequently evolve in real time. Therefore, a first step toward the goal of the adaptive learning system of optimizing the learning gain is tracing the learners' ability evolution in a fast and accurate manner.

In the context of intelligent tutoring systems (ITS), there are specialized approaches for tracing the learner's mastery of knowledge. A representative example is Bayesian knowledge tracing (BKT; Corbett and Anderson, 1994). In BKT, the learner's knowledge state is represented by a set of multiple binary latent variables that indicate mastery or non-mastery of the skills. The probability of having mastered each skill is estimated by binary measurement outcomes (correct or incorrect responses to items) and iteratively updated by using the rule of Bayes. Similar to CAT, however, the methods require a calibration on large samples using some nontrivial estimation techniques (expectation-maximization algorithm, or exhaustive search) that require high computational power (Papousek et al., 2014; Pálanek, 2016).

To that extent, an interesting alternative that can be considered for tracking the learner's ability evolution is the Elo rating system (ERS; Elo, 1978). The ERS was originally developed for calculating relative skill levels of players in chess performances, and the method also has widely been used in sport statistics for paired competitions (e.g., major league baseball). More recently, the ERS has been applied to various contexts of educational and psychological studies (e.g., Attali, 2014; Brinkhuis et al., 2015). In regard of its application to onlinelearning environment, the paired competition can be thought of as an interaction between the learner and the item. In general, the ERS algorithm is formulated to update the learner ability and item difficulty parameters from the Rasch model. To be specific, once a learner has responded to an item, the ERS updates the individual learner's ability level estimate that was based on his or her previous trajectory. Given the learner's current ability level, the next item is chosen by its difficulty level. A practical strength of this approach is that the method is conceptually fast and readily implementable in any software.

Several articles compared the performance of ERS with that of traditional IRT modeling to explore whether its parameter estimation is as accurate as the traditional approach. Maris and Van der Maas (2012) showed that the ability estimates updated from ERS method is highly correlated with the expected a posteriori (EAP) estimates from an IRT model when a speedaccuracy trade-off scoring rule was used. Studies also compared the performance of ERS with alternative methods for estimating item difficulties. For example, Wauters et al. (2012) compared the quality of the ERS-based item difficulty estimates with those based on maximum likelihood procedures, proportion correct, and human judgement methods, and found that the ERS provides reliable results with a sample size of 200 learners. Similarly, Pálanek (2016) provided evidence that there is a high correlation between ERS-based item difficulty estimates and joint maximum likelihood-based estimates.

Researchers (e.g., Klinkenberg et al., 2011; Savi et al., 2015; Braithwaite et al., 2016; Coomans et al., 2016; Hofman et al., 2018) also provided empirical evidence in favor of the ERS, by means of massive log data from Maths Garden, a CAP system where the learner ability and item difficulty levels are updated on the fly. Park et al. (2018) proposed a method to alleviate the cold-start in adaptive learning systems - the problem that for new learners we do not have an idea of their ability and therefore the adaptive learning environment might not perform well until the learner made a substantial number of items. The authors proposed using an explanatory IRT model based on learner-item interaction data and learner features (e.g., age, gender, or learning disability) and estimate the learners initial ability levels and their ability changes while not engaged in the learning environment.

Despite the increasing number of studies applying the ERS in adaptive learning systems, in the majority of these studies, the ERS is intended to track just a single broad ability. In contrast, monitoring multiple abilities not only forms the basis of learners' understanding of the material, but also provides direct information to educational researchers and instructors as to the areas that learners need to improve upon (FerriniMundy and Schmidt, 2005). Therefore, identifying his or her progress on more fine-grained ability dimensions would imply an important advancement of the adaptive learning system, because of the sheer amount of information about the learner's learning state. Doebler et al. (2015) and Pálanek (2016) proposed an improved ERS algorithm for tracking multiple dimensions of ability. Yet, their methodological focus is still on situations where items are allowed to load on only one of the multiple ability dimensions in the answering process. More recently, Chen et al. (2018) and Tang et al. (2018) used a Markov decision process to track multiple dimensions of ability. In these studies, the learner's ability was modeled by a set of multiple binary latent variables that indicate mastery or non-mastery of the skills while a reinforcement learning approach was proposed to recommend personalized items.

In the current article, therefore, we propose to address these issues by using a multidimensional IRT (MIRT) model to track the (continuous) ability parameter estimates within ERS. The basic idea is that instead of assuming a unidimensional trait of item responses, our approach will assume that a single item may involve more than one ability parameters. Therefore, we extend the standard ERS that updates a single ability parameter based on the Rasch model, and will allow to have a simultaneous update on multiple ability parameters based on a compensatory MIRT model ("M-ERS").

In the next section we give more details on the methodological framework of the ERS and its application to educational settings. We then propose our method ("M-ERS") that is formulated to update multiple abilities. Next, we will evaluate the performance of our method through three simulation studies. Furthermore, 
the method will be demonstrated using a real application of learning data obtained from an educational platform for children's math ability development. We end with conclusions and implications.

\section{ELO RATING SYSTEM}

The ERS is originally rooted in the Bradley Terry Luce (BTL; Bradley and Terry, 1952) model, a probabilistic model that predicts the outcome of players in a type of paired competitions. Specifically, the expected outcome that one player defeats his or her opponent is formulated as follows:

$$
P_{i j}=P(i \text { defeats } j)=\frac{\theta_{i}}{\theta_{i}+\theta_{j}},
$$

where $\theta_{i}$ and $\theta_{j}$ represent the ratings (e.g., latent traits) of players $i$ and $j$, respectively. By setting up $\theta_{i}=e^{\theta_{i}}$, Equation (1) can be transformed to a logistic function of the difference between $\theta_{i}$ and $\theta_{j}$, which comprises of the expected outcome of the ERS. That is,

$$
P_{i j}=P(\text { i defeats } j)=\frac{\exp \left(\theta_{i}-\theta_{j}\right)}{1+\exp \left(\theta_{i}-\theta_{j}\right)}
$$

Likewise, both the BTL model and the ERS are based on the probability of winning a competition; however, the latter method is additionally intended to supply easy-to-compute updates as new outcomes are observed. In other words, the ERS takes an algorithmic heuristic to easily update the expected outcome for the next iteration, based upon the estimated latent trait (i.e., $\theta_{i}$ and $\theta_{j}$ ) at the current iteration. Kiraly and Qian (2017) showed that the derivative of a likelihood function for Equation (2) based on a single data point produces the following updating component for the ERS algorithm:

$$
\frac{\partial l\left(\theta_{i}, \theta_{j} \mid Y_{i j}\right)}{\partial \theta_{i}}=Y_{i j}\left(1-P_{i j}\right)-\left(1-Y_{i j}\right) P_{i j}=Y_{i j}-P_{i j},
$$

where $l\left(\theta_{i}, \theta_{j} \mid Y_{i j}\right)=Y_{i j} \log P_{i j}+\left(1-Y_{i j}\right) \log \left(1-P_{i j}\right)$.

In sum, given the observations for a competition between players $i$ and $j$, the estimates of $\theta_{i}$ and $\theta_{j}$ are updated simultaneously. Specifically,

$$
\begin{gathered}
\hat{\theta}_{i}=\hat{\theta}_{i}+K\left\{Y_{i j}-P_{i j}\right\} \text { for a player } \mathrm{i}, \\
\hat{\theta}_{j}=\hat{\theta}_{j}-K\left\{Y_{i j}-P_{i j}\right\} \text { for a player } \mathrm{j} .
\end{gathered}
$$

In the equation above, the term $\left\{Y_{i j}-P_{i j}\right\}$ can be viewed as the discrepancy between what is expected and what is observed. In fact, the ERS can be viewed as a type of the stochastic gradient descent (SGD; Robbins and Monro, 1951) algorithm where the updating rule in the system corresponds to the update of parameters along the error gradient (Pálanek, 2016). The update will be larger if the current parameter setting produces a large discrepancy. Note that $K$ is a step size that defines to what extent the ability estimate can be affected by the difference between the current and expected responses for the student $p$.

\section{APPLICATION TO ADAPTIVE LEARNING SYSTEMS}

In adaptive learning environments, the paired competition occurs when the learner interacts with the learning material (=item). The ERS process can be applied as follows. Consider $\theta_{i(t)}$ be an ability of a learner $i$ (unidimensional continuous variable) after solving an item at measurement occasion $t$. Also, suppose $Y_{i j(t)}$ be the learner $i$ outcome for item $j$ measured at measurement occasion $t$, where the outcome is dichotomously scored $(0=$ incorrect; $1=$ correct answer to the item). Then the ERS for updating the ability parameter takes the following sequence:

$$
\begin{aligned}
& \hat{\theta}_{i(t)}=\hat{\theta}_{i(t-1)}+K\left\{Y_{i j(t)}-P_{i j(t)}\right\} \text { for a learner } \mathrm{i} \\
& \hat{\beta}_{j(t)}=\hat{\beta}_{j(t-1)}-K\left\{Y_{i j(t)}-P_{i j(t)}\right\} \text { for an item } \mathrm{j},
\end{aligned}
$$

where $\hat{\theta}_{i(t-1)}$ is the ability estimate at the previous measurement time $t-1$ for the learner $i, \hat{\beta}_{j(t-1)}$ is the item difficulty estimate at the previous measurement time $t-1$ for the item $j$, and $P_{i j(t)}$ is the expected response for the current measurement occasion $t$. Consequently, a learner interacting with a very difficult item risks losing a little bit of ability level in case of failure, with the possibility of gaining much greater ability level in case of success. Several studies have explored the optimal step size $K$ for the ERS for student modeling. Wauters et al. (2012) suggested using a constant step size, $K=0.4$ in the context of educational data. On the other hand, other studies (e.g., Glickman, 1999; Klinkenberg et al., 2011; Papousek et al., 2014; NiŽnan et al., 2015) proposed that the step size needs to decrease as a function of a total number of item answered and therefore the system gains more information about the learner's true ability level.

In Equation (5), it is possible that the outcome $Y_{i j(t)}$ can be scored by considering whether the learner completed the item within the allotted limit. Maris and Van der Maas (2012) derived a scoring rule that accounts for response time and accuracy, and applied it within ERS. While the ERS can be used to gradually obtain reliable estimates of both student's abilities and item difficulties, adaptive item sequencing can be more efficient if we could start from a pre-calibrated item bank, including information on item difficulty and possibly other characteristics of items, and from which items with undesired characteristics are excluded (van Groen et al., 2014). In this case, $\hat{\beta}_{j(t)}$ in Equation (5) needs not be updated.

\section{MULTIDIMENSIONAL EXTENSION OF THE ERS (M-ERS)}

In this section we propose an extended version of the ERS that enables the system to track multidimensional abilities in real time. Specifically, the proposed algorithm can handle two types of dimensional structures in the item bank-(a) "within-item dimensionality" where a single item can be associated with more than one task ability; as well as (b) "between-item dimensionality" where a set of items is associated with multiple abilities, while each item measures only one of those abilities. 
Suppose an adaptive learning environment contains an item bank that is designed to measure a total of $M$-dimensional abilities i.e., $\theta_{i}=\left(\theta_{i 1}, \ldots, \theta_{i M}\right)^{\prime}$ for a student $i$. The multidimensional dichotomous logistic model (Reckase, 1985) that describes the probability of a correct answer to item $j$ can be formulated by either conjunctive or compensatory assumptions about how the latent abilities are combined. In a conjunctive model assumption, it is assumed that the learner should have each of the relevant abilities in order to answer an item correctly. The probability of a correct response therefore is a joint product of the inverse logit function of the difference between each of the abilities and the corresponding item difficulty:

$$
P_{i j}=P\left(Y_{i j}=1\right)=\prod_{m=1}^{M} \frac{\exp \left(\alpha_{j m}\left[\theta_{i m}-\beta_{j}\right]\right)}{1+\exp \left(\alpha_{j m}\left[\theta_{i m}-\beta_{j}\right]\right)},
$$

where $P\left(Y_{i j}=1\right)$ indicates the probability of a correct answer, $\theta_{i m}$ is the $m$ th ability parameter of the learner $i(m=1, \ldots, M)$, $\alpha_{j m}$ is the item discrimination of the item $j$ corresponding to $m$ th ability dimension, and $\beta_{j}$ denotes the overall difficulty level of the item $j$. On the other hand, in a compensatory model, on the other hand, it is assumed that the lack of one ability can be compensated by greater level of another ability, as follows:

$$
P_{i j}=P\left(Y_{i j}=1\right)=\frac{\exp \left(\sum_{m=1}^{M} \alpha_{j m} \theta_{i m}-\beta_{j}\right)}{1+\exp \left(\sum_{m=1}^{M} \alpha_{j m} \theta_{i m}-\beta_{j}\right)} .
$$

The difference between the observed and the expected performance $P_{i j}$ based on the multidimensional IRT models is used to update the ability parameters after each item response. Specifically, the $P_{i j}$ within ERS for the $m$-th ability for person $i$ on measurement occasion $t$ is updated as follows:

$$
\begin{aligned}
\hat{\theta}_{i m(t)} & =\hat{\theta}_{i m(t-1)}+D_{m(t)} K\left\{Y_{i j(t)}-P_{i j(t)}\right\} \\
\hat{\beta}_{j(t)} & =\hat{\beta}_{j(t-1)}-D_{m(t)} K\left\{Y_{i j(t)}-P_{i j(t)}\right\},
\end{aligned}
$$

where $D_{m(t)}$ is a weight to specify whether the $m$ th ability is indicated by the item given at $t$-th step. For the ability that is indicated by the item, $D_{m(t)}$ equals 1 . For the ability that is not indicated by the item, the weight takes values between zero and one.

\section{SIMULATION STUDY}

To explore the performance of the M-ERS method in terms of estimating the real-time evolution of multidimensional ability parameters for individual learners, we apply the method to data generated under a variety of conditions. In accordance with our research questions, the simulation study consists of three parts. In Study 1, we examine the result of a standard ERS that naively assumes the unidimensionality of ability parameter, where in fact data involve a multidimensional ability. In Study 2, we explore the performance of the M-ERS in relation to the total number of items answered. In Study 3, we investigate the performance of the modified M-ERS in which both response time and accuracy data

\begin{tabular}{|c|c|c|c|c|c|c|c|}
\hline \multirow[t]{2}{*}{ Item ID } & \multirow[t]{2}{*}{$\beta_{\mathbf{j}}$} & \multicolumn{3}{|c|}{ Item bank 1} & \multicolumn{3}{|c|}{ Item bank 2} \\
\hline & & $\theta_{1}$ & $\theta_{2}$ & $\theta_{\mathbf{3}}$ & $\theta_{1}$ & $\theta_{2}$ & $\theta_{3}$ \\
\hline 1 & -2.534 & 1 & . & . & 1 & . & . \\
\hline 2 & -2.21 & 1 & . & . & 1 & . & . \\
\hline 3 & 1.326 & 1 & . & . & 1 & . & . \\
\hline 4 & 0.253 & 1 & . & . & 1 & . & . \\
\hline 5 & 1.275 & 1 & . & . & 1 & . & . \\
\hline 6 & 0.089 & 1 & . & . & 1 & . & . \\
\hline 7 & -0.001 & 1 & 1 & . & . & 1 & . \\
\hline 8 & -1.256 & 1 & 1 & . & . & 1 & . \\
\hline 9 & 2.242 & 1 & 1 & . & . & 1 & . \\
\hline 10 & -1.556 & 1 & 1 & . & . & 1 & . \\
\hline 11 & 2.213 & 1 & 1 & . & . & 1 & . \\
\hline 12 & -3.3 & 1 & . & 1 & . & . & 1 \\
\hline 13 & 0.753 & 1 & . & 1 & . & . & 1 \\
\hline 14 & -2.246 & 1 & . & 1 & . & . & 1 \\
\hline 15 & -1.156 & 1 & . & 1 & . & . & 1 \\
\hline
\end{tabular}
are incorporated.
TABLE 1 | Patterns of multidimensionality (a sample of 15 items from two item banks).

"1" indicates that the item loads on the dimension.

\section{Item Bank}

Following the literature on the MIRT (e.g., Adams et al., 1997; Hartig and Höhler, 2008), we consider two loading structures to determine patterns of the multidimensionality. Specifically, two types of item banks are created- (a) when items are allowed to load on more than a single ability dimension ("Item bank 1"); and (b) when items are allowed to load on only one of the multiple ability dimensions ("Item bank 2"). Each of them includes 200 operational items, measuring a total of three dimensions. Item bank 1 consists of a primary dimension $\theta_{1}$ indicated by all the items, and two auxiliary dimensions, $\theta_{2}$ and $\theta_{3}$, indicated by $35 \%$ of items and the $25 \%$ of the remaining items. In Item bank 2 , each item involves only one out of the three dimensions. Specifically, $40 \%$ of items involves the 1 st dimension, $35 \%$ of them involves the 2 nd dimension, and the remaining $25 \%$ of them involves the 3rd dimension (as an illustration, Table 1 gives a sample of 15 items from two item banks). Based on each item bank, data are generated under a compensatory IRT model with difficulty and discrimination parameters, mimicking realistic test items:

$$
P_{i j}=P\left(Y_{i j}=1\right)=\frac{\exp \left(\sum_{m=1}^{3} \alpha_{m j} \theta_{j m}-\beta_{j}\right)}{1+\exp \left(\sum_{m=1}^{3} \alpha_{m j} \theta_{j m}-\beta_{j}\right)},
$$

where the generating parameter values for the difficulty and the slope parameters are drawn from $\beta_{j} \sim N(0,1)$ and $\alpha_{m j} \sim$ $U(0.5,2)$, where $j=1, \ldots, 200$ (items) and $m=1,2,3$ (dimensions of ability).

\section{Persons}

A total of $n=250$ learners are considered in the simulation studies. The population distribution of ability parameters is taken to be $N(\mu, \Sigma)$, where $\mu=(1,1,1)^{\prime}$. In $\Sigma$, all variances are equal to 
1 and the three bivariate correlations are equal: dimensions were independent $\left(\rho_{m m^{\prime}}=0.0\right)$, weakly correlated $\left(\rho_{m m^{\prime}}=0.2\right)$, or moderately correlated $\left(\rho_{m m^{\prime}}=0.5\right)$.

A total of 6 data sets were generated by following 6 scenarios ( 2 patterns of dimensionality $\times 3$ correlations among ability dimensions). In each condition, the M-ERS method will be used to update the three ability parameter estimates for each learner as he or she attempts on a sequence of items. We assume that each learner is assigned a sequence of 200 items that are randomly selected. That is, item sequences are varied across learners. In M-ERS, in Equation (7), the expected response is estimated by using a compensatory IRT model with a constraint that the slope parameters $\alpha_{m j}$ 's are equal to 1 for simplicity (the inclusion of different loadings is a logical further extension). That is,

$$
P\left(Y_{i j(t)}=1\right)=\frac{\exp \left(\sum_{m=1}^{3} \theta_{j m}-\beta_{j}\right)}{1+\exp \left(\sum_{m=1}^{3} \theta_{j m}-\beta_{j}\right)} .
$$

In the equation, we use step size $\mathrm{K}$ that linearly decreases as a function of a total number of items answered between the maximum value of 0.4 and the minimum value of 0.1 . Note that we assume that item difficulty parameters are considered as known (based on a calibration study), and the difficulty estimates therefore will not be further updated within the M-ERS.

\section{STUDY 1: PREDICTION ACCURACY OF A STANDARD ERS AND M-ERS}

In the first study, we explore the extent to which the unidimensional ability assumption embedded in a standard ERS has an impact on the prediction accuracy (in terms of the learners' future responses), when the truth is that the responses to the learning items involve three-dimensional ability parameters. As seen in Equation (8), predictions of the responses of learners can be achieved by using the ERS algorithm based on the known item parameters and the learner ability estimate(s) predicted by the preceding step. The prediction accuracy is calculated by classifying the expected response $\left(=P_{i j(t)}\right)$ with a certain cut-point into the observed response $\left(=Y_{i j(t)}\right)$ on measurement occasion $t$. To evaluate the quality of predictions, we use a Receiver Operating Characteristic curve (ROC). The ROC curve represents the relation between true positive rates $[=\mathrm{TP} /(\mathrm{TP}+\mathrm{FN})]$ and false positive rates $[\mathrm{FP} /(\mathrm{FP}+\mathrm{FN})]$ at various probability cut-off points. In case of totally random predictions, the Area Under Receiver Operating Characteristic curve (AUROC) is approximately equal to 0.5 .

Figure 1 visualizes the ROC curves, comparing the performances of a standard ERS and the M-ERS. Each panel in the figure includes 6 curves, representing a combination of simulation conditions (2 patterns of dimensionality $\times 3$ correlations among ability parameters). The $\mathrm{x}$-axis and $\mathrm{y}$-axis indicate the false positive rate and the true positive rate. Note that the best possible prediction method would yield a point in the upper left corner or coordinate $(0,1)$ of the ROC space, representing $100 \%$ sensitivity and specificity. Therefore, results from the two panels suggest that M-ERS outperforms the

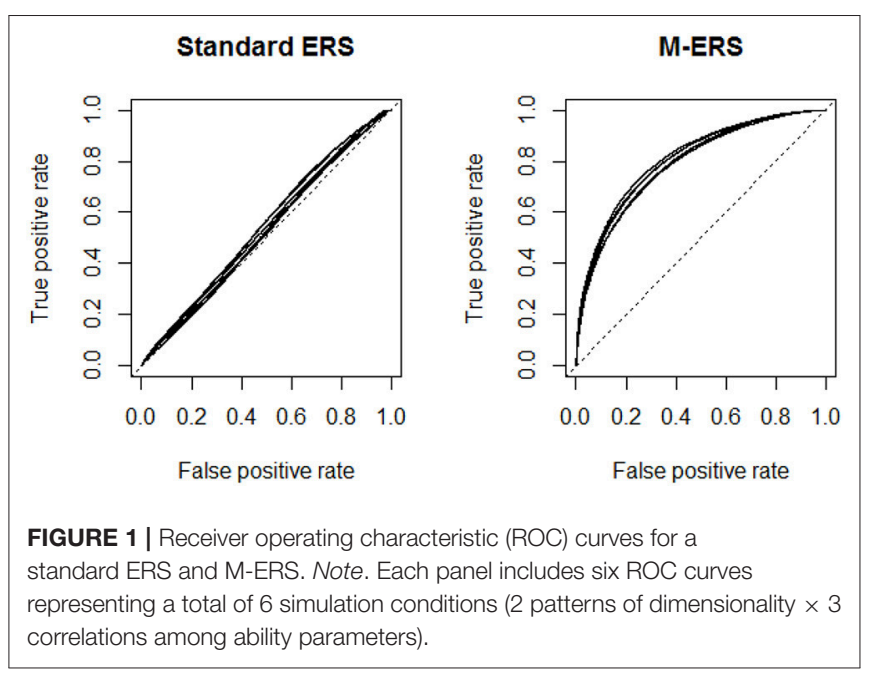

TABLE 2 | Area Under ROC curve (AUROC) for a standard ERS and M-ERS.

\begin{tabular}{lccccc}
\hline & \multicolumn{2}{c}{ Item bank 1 } & & \multicolumn{2}{c}{ Item bank 2 } \\
\cline { 2 - 3 } \cline { 5 - 6 } \cline { 5 - 6 } & Standard ERS & M-ERS & & Standard ERS & M-ERS \\
\hline$\rho=0.0$ & 0.5197 & 0.8038 & & 0.5216 & 0.7850 \\
$\rho=0.2$ & 0.5204 & 0.8070 & & 0.5324 & 0.7900 \\
$\rho=0.5$ & 0.5496 & 0.8157 & & 0.5494 & 0.7893 \\
Average & 0.5299 & 0.8088 & & 0.5345 & 0.7881 \\
\hline
\end{tabular}

standard ERS in all six simulated scenarios. Table 2 summarizes the area under the ROC curves (AUROC). In case of M-ERS, the AUROCs are much higher than the expected values using random predictions (i.e., 0.5 for AUROC). It is seen that the AUROCs for M-ERS are 0.8088 and 0.7881 for Item banks 1 and 2, respectively. However, the standard ERS generally reveals only performs marginally better than the random predictions (i.e., 0.5299 for Item bank 1 and 0.5345 for Item bank 2).

\section{STUDY 2: ABILITY PARAMETER ESTIMATION AS A FUNCTION OF NUMBER OF ITEMS ANSWERED}

In the second study, we investigate the performance of $\mathrm{M}$ ERS as a function of the total number of items answered. We also examine the effect of different simulation conditions (i.e., dimensionality patterns and correlations among true three ability parameters) on the ability parameter estimation. To evaluate the quality of the ability estimation, the estimated ability parameters are summarized by mean squared error (MSE). In particular, the differences between the true and estimated abilities at measurement occasion $t$ are squared, and averaged over the entire sample size of new students. That is, for the learner $i$ at the measurement occasion $t$ :

$$
\operatorname{MSE}\left(\hat{\theta}_{m(t)}\right)=\frac{\sum_{i=1}^{N}\left(\hat{\theta}_{i m(t)}-\theta_{i m(t)}\right)^{2}}{N}
$$



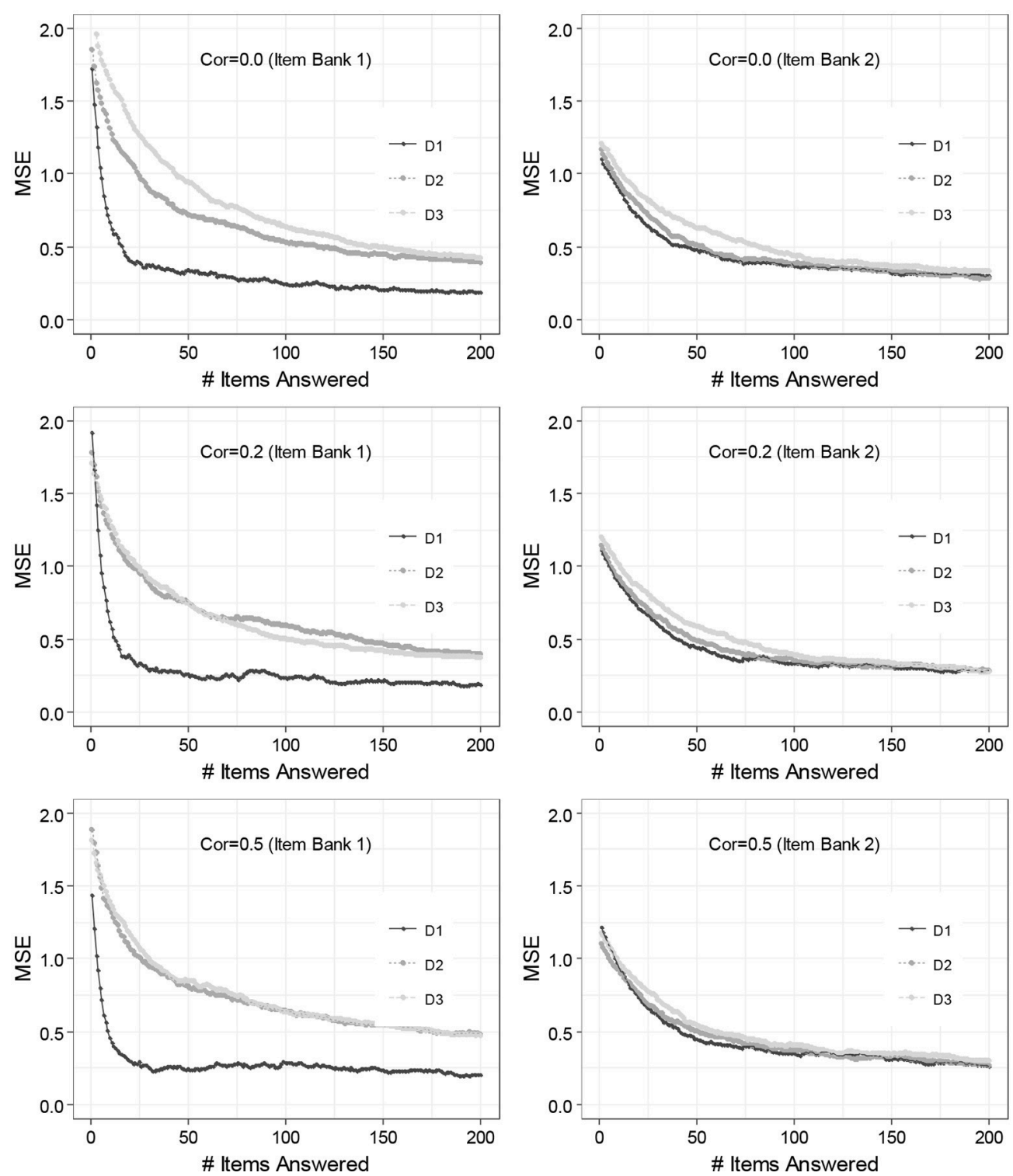

FIGURE 2 | Result of 3-dimensional ability estimation across the number of items answered. Cor, correlations between dimensions; D1, 1st dimension; D2, 2nd dimension; D3, 3rd dimension.

Figure 2 includes line plots demonstrating the performances of the M-ERS for two different patterns of dimensionality. Each panel in the figure represents a different simulation condition with the patterns of dimensionality (Item banks 1 and 2) and the true correlations among ability parameters $\left(\rho_{\mathrm{mm}^{\prime}}=0.0\right.$, 0.2 , and 0.5 ), with a total number of items answered being on the $\mathrm{x}$-axis and the MSEs on the $\mathrm{y}$-axis. The three lines in each panel comprise the squared difference between the $\hat{\theta}_{i m(t)}$ and $\theta_{i m(t)}$ averaged across $n=250$ individual learners for the three dimensions ("D1," "D2," and "D3" in the legend). Remind that the three panels on the left-hand side summarize the performance of the M-ERS method for Item bank 1 that exhibits one primary dimensions plus two auxiliary dimensions (Item bank 1). The other three on the right-hand side are based on the data where each item involves only a single dimension. See the section of "Study Design" for more details.

Overall, results suggest that that the MSEs tend to decrease as the total number of items answered increases. The finding is common to all three dimensions ("D1," "D2," and "D3"), but the speed of decrease varies by the number of items the ability dimension involves. For Item bank 1 (column left), it is seen that MSE for the 1st dimension (on which all items load) reveals a dramatic decrease while the first 20 items are answered (to around 0.2). Similarly, though more gradually, MSEs for 2nd and 3rd dimensions (on which only 35 and 25\% of the items load) also tend to decrease. In these two auxiliary 
dimensions, however, the MSE does not reach 0.2, even up to 200 items.

For Item bank 2 (column right), on the other hand, the degrees of the decreasing trends are extremely similar among the three dimensions. This can be explained by the fact that the three dimensions involve a similar amount of items i.e., 40, 35 , and $25 \%$ of items load on the D1, D2, and D3, respectively. For moderate scenarios, in particular, the difference becomes extremely tiny. It is seen that the true correlations among ability parameters do not have an impact on MSEs across any measurement occasions.

As an alternative way to check the performance of M-ERS, Figure 3 compares the ability estimates of 250 learners after 200 Elo-updates with the expected a posteriori (EAP) ability estimates obtained by fitting a compensatory IRT approach. Overall, ability estimates from EAP and M-ERS are highly correlated, regardless of any simulation conditions and the ability dimensions. The correlation coefficients $(3$ dimensions $\times 2$ item banks $\times 3$ correlation among true abilities) range from 0.967 to 0.990 . Note that the EAP estimates are the results of an analysis that requires responses of many persons on many items, and is computing-intensive and therefore cannot be used on the fly. Therefore, the EAP estimates are used here as a benchmark, but they cannot be considered as a viable alternative for the ERS approach.

\section{STUDY 3: M-ERS FOR SPEED-ACCURACY TRADEOFF}

There have been an increasing number of studies (e.g., Tuerlinckx and De Boeck, 2005; van der Linden, 2007; De Boeck et al., 2017) that account for response time as well as response accuracy in order to model the ability parameter. Of several statistical and psychological approaches to the response time modeling (van der Linden, 2009), one of the promising methods is to model response time and accuracy from the measurement perspective by two-step procedures; specifically, (a) setting up a scoring rule and (b) fitting a proper statistical model that conforms scores of the type. Klinkenberg et al. (2011) showed that the ERS method outperforms a standard CAT method (specifically, Eggen and Verschoor, 2006) when the speed-accuracy tradeoff scores [so called high speed high stake (HSHS)] and the corresponding model were used. We do not know studies that model multidimensional ability trajectories based on both response time and accuracy within ERS. Therefore, we aim to explore the incorporation of the HSHS scoring rule in the proposed M-ERS method.

According to the HSHS scoring rule, the observed scores can be calculated as follows: $S_{i j}=\left(2 Y_{i j}-1\right)\left(d-T_{i j}\right)$, where $Y_{i j}$ is an accuracy for the learner is response to the item $j, d$ is a time limit, and $T_{i j}$ is an time spent for the learner $i$ until answering the item $j$. In this expression the residual time i.e., $\left(d-T_{i j}\right)$ can compensate or penalize for the learner, corresponding to the learner's accuracy to the item. In particular, for a correct response (i.e., $Y_{i j}=1$ ), the learner will gain the residual time as a score. Similarly, for an incorrect response (i.e., $Y_{i j}=0$ ), the score will
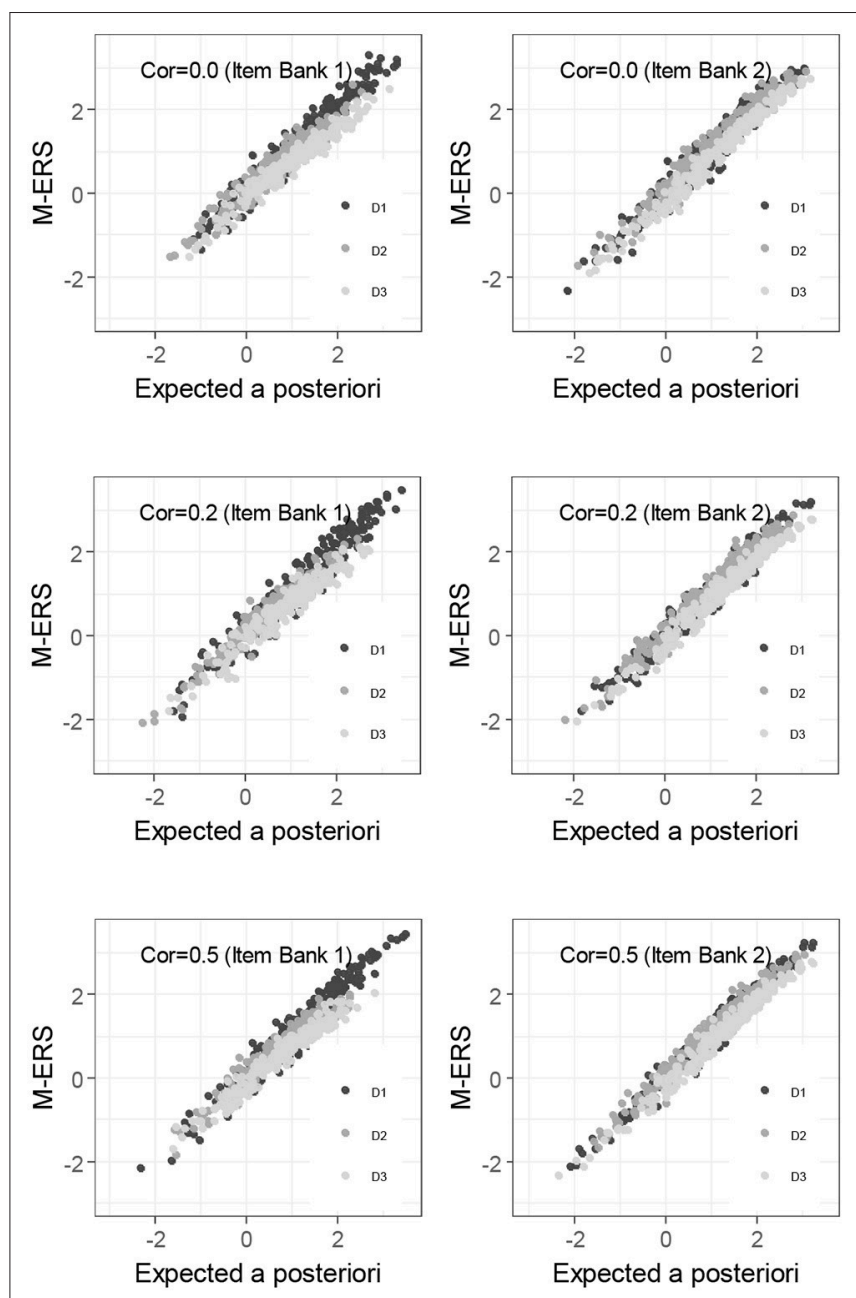

FIGURE 3 | Relations between EAP and M-ERS estimates.

be reduced by the same amount. In current study, the maximum time given each item $(=d)$ is restricted to be 1 , so the residual time simply reflects the proportion of time left. Such a scoring scheme is especially useful to control for the case that the learner guesses instantaneously guess the given item (a quick incorrect answer). The expectation of the trade-off score, $E\left(S_{i j}\right)$, for an item that is based on three abilities can naturally be extended from a unidimensional version in Maris and Van der Maas (2012). That is,

$E\left(S_{i j}\right)=\frac{\exp \left(2\left(\sum_{m=1}^{3} \theta_{i m}-\beta_{j}\right)\right)+1}{\exp \left(2\left(\sum_{m=1}^{3} \theta_{i m}-\beta_{j}\right)\right)-1}-\frac{1}{\left(\sum_{m=1}^{3} \theta_{i m}-\beta_{j}\right)}$,

where $m=1,2,3$ (abilities). Specifically, the $E\left(S_{i j}\right)$ in Equation (10) provides the expected HSHS score for learner is to solve the item $j$.

We conduct a simulation study to compare this approach with the M-ERS method based on the accuracy data only. The 
TABLE 3 | Comparing IRT-based ERS algorithms for correctness vs. trade-off score (correctness and speediness combined).

\begin{tabular}{|c|c|c|c|c|c|c|c|}
\hline & & \multicolumn{2}{|c|}{$\rho=\mathbf{0 . 0}$} & \multicolumn{2}{|c|}{$\rho=\mathbf{0 . 2}$} & \multicolumn{2}{|c|}{$\rho=\mathbf{0 . 5}$} \\
\hline & & |Bias| & MSE & |Bias| & MSE & |Bias| & MSE \\
\hline \multicolumn{8}{|c|}{ ITEM BANK 1} \\
\hline \multirow[t]{2}{*}{$\hat{\theta}_{1}$} & Accuracy & 0.009 & 0.300 & 0.008 & 0.286 & 0.009 & 0.289 \\
\hline & Speed-accuracy & 0.008 & 0.245 & 0.008 & 0.232 & 0.007 & 0.213 \\
\hline \multirow[t]{2}{*}{$\hat{\theta}_{2}$} & Accuracy & 0.010 & 0.364 & 0.009 & 0.363 & 0.010 & 0.382 \\
\hline & Speed-accuracy & 0.009 & 0.306 & 0.009 & 0.298 & 0.009 & 0.307 \\
\hline \multirow[t]{2}{*}{$\hat{\theta}_{3}$} & Accuracy & 0.010 & 0.400 & 0.010 & 0.395 & 0.010 & 0.392 \\
\hline & Speed-accuracy & 0.009 & 0.337 & 0.009 & 0.336 & 0.009 & 0.332 \\
\hline \multicolumn{8}{|c|}{ ITEM BANK 2} \\
\hline \multirow[t]{2}{*}{$\hat{\theta}_{1}$} & Accuracy & 0.008 & 0.291 & 0.008 & 0.294 & 0.008 & 0.290 \\
\hline & Speed-accuracy & 0.008 & 0.245 & 0.008 & 0.251 & 0.008 & 0.252 \\
\hline \multirow[t]{2}{*}{$\hat{\theta}_{2}$} & Accuracy & 0.008 & 0.299 & 0.008 & 0.290 & 0.008 & 0.291 \\
\hline & Speed-accuracy & 0.008 & 0.260 & 0.008 & 0.245 & 0.008 & 0.252 \\
\hline \multirow[t]{2}{*}{$\hat{\theta}_{3}$} & Accuracy & 0.009 & 0.337 & 0.009 & 0.353 & 0.009 & 0.315 \\
\hline & Speed-accuracy & 0.008 & 0.299 & 0.009 & 0.310 & 0.008 & 0.278 \\
\hline
\end{tabular}

|Bias|, absolute value of the bias averaged over learners and items; MSE, a mean squared error averaged over learners and items.

entire data-generating process follows what is described in the section "Study Design." The response time data is generated for each learner who solves each item, using a formula for expected response time from Maris and Van der Maas (2012), where the time limit for each item is consistently set at 1-min. Like in studies 1 and 2, the simulation conditions are combinations of patterns of dimensionality (Item banks 1 and 2) and the true correlations among ability parameters $\left(\rho_{m m^{\prime}}=0.0,0.2\right.$, and 0.5$)$.

Table 3 comprises two tables that compare the performance of two M-ERS method when only accuracy data are used ("Accuracy") or both speed and accuracy data ("SpeedAccuracy") are used. Overall results suggest that M-ERS for speed-accuracy data shows smaller MSE, regardless of any simulation conditions.

\section{REAL DATA ANALYSIS}

\section{Description}

For illustrative purposes, we used a dataset collected from a webbased learning platform, "Number Sense" (Linsen et al., 2016) developed by KU Leuven, Belgium. It was designed as an itembased e-learning environment for 6- to 8-year-old children and includes approximate number discrimination tasks, symbolic comparison tasks, and symbolic and non-symbolic number line estimation tasks. In particular, current data were collected between Fall 2017 and Spring 2018, during one school year. It includes data from 299 students' responses to 330 items in total. Among the items, 168 of them are designed to measure (a) comparison ability and the remaining 162 items are designed to measure (b) number line estimation ability. There were no items that require both. All responses to the items are scored for accuracy i.e., the binary scale (correct/incorrect). Current log data do not include response times.
For the purpose of obtaining item parameters, data from 200 out of 299 students were used to fit a MIRT formula i.e., $P\left(Y_{i j}=1\right)=\frac{\exp \left(\theta_{j 1}+\theta_{j 2}-\beta_{j}\right)}{1+\exp \left(\theta_{j 1}+\theta_{j 2}-\beta_{j}\right)}$, where $\theta_{i 1}$ and $\theta_{i 2}$ reflect abilities in relation to the comparison and number line estimation, respectively. The remaining 99 students were used to illustrate the Elo algorithm. For the estimation procedure with this training set, the MCMC algorithm is implemented with R 3.3.3 (R Core Team, 2013). More specifically, JAGS (Plummer, 2015) was implemented by an R package "R2jags" (Su and Yajima, 2015) that provides wrapper functions for the Bayesian analysis program. For each analysis with the JAGS, four chains were run, and each ran for 10,000 iterations. We used a thinning parameter of four and used the first half as burn-in. (Gelman and Rubin's, 1995) statistics are used for a convergence diagnostic. Results of the Bayesian inference show that the posterior predictive mean for the correlation between the comparison and number line estimation abilities (i.e., $\theta_{i 1}$ and $\theta_{i 2}$ ) was approximately $\hat{\rho}=$ 0.13 . The posterior predictive means for item discriminations and difficulties (i.e., $\hat{\alpha}_{m j}$ and $\hat{\beta}_{j}$ ) were used as known item parameters, and where therefore not updated within the Elo algorithm.

\section{Results}

Figure 4 shows the resulting ability trajectory of two randomly chosen students by fitting a standard ERS and the M-ERS. As in the simulation result, the figure presents the impact of assuming unidimensionality or multidimensionality of the ability parameter. It is noticeable that the ability estimates obtained by a standard ERS are in general greater than the two-dimensional ability estimates from the M-ERS. Based on the similarity of the Number Sense data to the data we generated in the simulation and the results we found there, we can suspect that the unidimensional ERS ability estimates for the Number Sense items are biased (upward), and the M-ERS has removed the bias. That implies that ignoring the multidimensional data structure may cause considerable bias in ability trajectory estimation in the learning environment, and therefore in a suboptimal adaptivity of the learning environment. After a longer sequence of items in the session, however, it is shown that the gaps among four approaches tend to be negligible.

\section{CONCLUSION}

In this paper, we have proposed an MIRT-based ERS method to address a dynamic estimation of the learner's progress in an adaptive practice environment where the learning items exhibit a multidimensional ability criteria. The model combines the idea of using a compensatory MIRT model to predict the learner performance with a fast and heuristic algorithm for tracking his or her irregular trend of ability parameters through the ERS.

First, we have shown that there occurs a considerable error in terms of updating the ability changes, when a unidimensional IRT is used in ERS when the truth is that there is a multidimensionality in a set of items. We have shown that the error in estimating the ability parameters can be alleviated with the compensatory IRT in ERS. Second, we have shown that at the initial step of learning, the error of ability estimates are bigger 


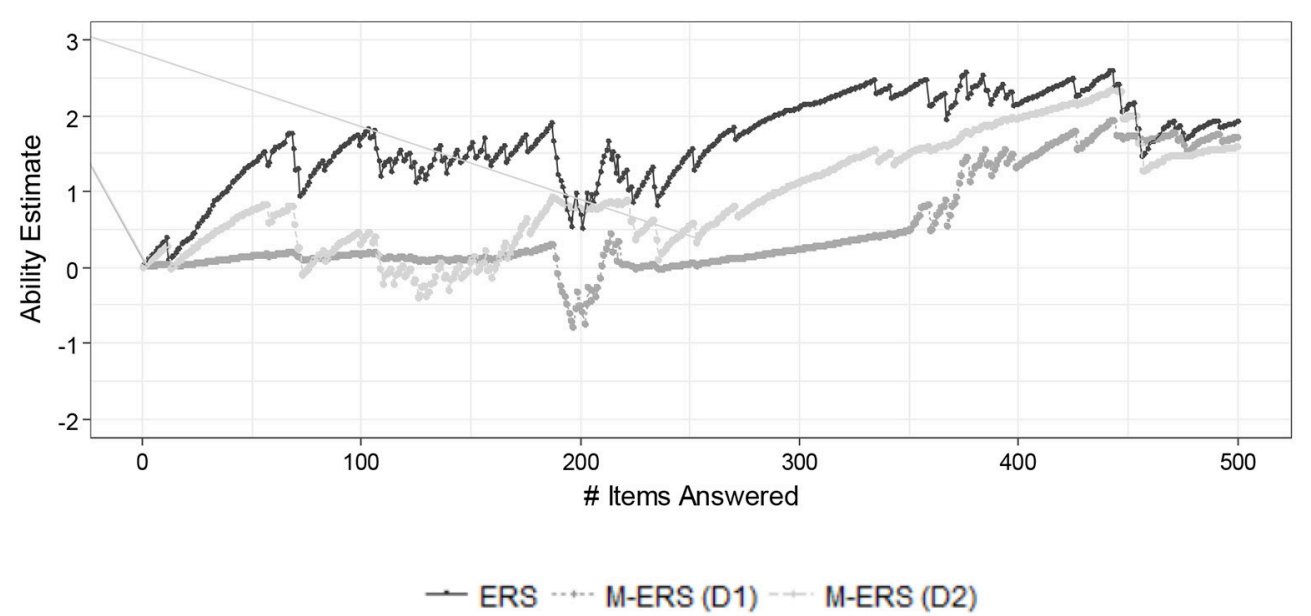

FIGURE 4 | Example of ability estimates for a student by standard ERS and M-ERS.

where each individual item involves more than one dimensions, as compared to the case where the item purely involves one of the multiple dimensions. However, we have found that the error has been noticeably reduced as more items are rendered. Third, we have extended the M-ERS method for the trade-off scores between response time and accuracy. Results show that bias and MSE of ability estimates are smaller when the HSHS (i.e., the speed-accuracy trade-off scoring that gives penalty for guessing) was incorporated in M-ERS than using the accuracy data only.

We believe that our approach offers the possibility to improve adaptivity when applied in an adaptive environment. In our simulation study, the items were chosen randomly across measurement occasions for each student, but it is also possible to administer items that optimize the item selection criteria. For example, the item can be chosen such that its difficulty level is as close as possible to the learner's current ability (e.g., 50\% chance of answering correctly). In the ERS based on the IRT formula, such an item selection strategy can be flexibly adjusted to avoid too easy (e.g., 90\% chance of answering correctly) or too hard (e.g., 25\% chance of answering correctly) items to individual learners. That means that the ERS can provide a flexible item sequencing tool for adaptivity in which a series of items are updated in real time based on their ability or knowledge levels (Wauters et al., 2010).

Another idea that may arise when considering to deal with ability estimation in ERS is to handle the cold-start problem i.e., the system does not know a new learner's ability level in the beginning of learning stage, when the new learner comes into the e-learning system for the first time. The cold start problem may also occur when a learner leave the e-learning system for a while and return (i.e., between-session period) because external effects could lead to the ability level change. Finally, the current simulation study shows a few limitations. For instance, the true ability was assumed to be constant over time, although it tends to evolve in learning environments. Including a time trend can add additional challenges, such as the determination of a step size that is large enough to keep track of the evolving ability but not too large in order to avoid very instable ability estimates.

Nevertheless, we believe the results of current study provide valuable information about how to efficiently follow up estimate multidimensional ability changes in the e-learning environments in order to alleviate concerns about the ERS and catalyze the usefulness of the e-learning system in educational settings.

\section{DATA AVAILABILITY}

The datasets generated for this study are available on request to the corresponding author.

\section{AUTHOR CONTRIBUTIONS}

JP developed the core ideas of the manuscript, the method, the design of the simulation study, as well as the real data analysis. WV supervised the entire work and gave feedback. FC and HvdM gave feedback on the results and the writing and contributed to the final manuscript.

\section{FUNDING}

This research includes a methodological approach and a real data example from the LEarning analytics for AdaPtiveSupport (LEAPS) project, funded by imec (Kapeldreef 75, B-3001, Leuven, Belgium) and the Agentschap Innoveren \& Ondernemen. The LEAPS project aimed to develop a self-learning analytical system to enable adaptive learning. This support system can be integrated into educational games and in supporting software for difficult readers and forprofessional communication. Partners from a broad field of expertise work together within the consortium. Examples include educational and cognitive scientists, software developers, statisticians, experts in humancomputer interaction and educational publishers. This extensive interdisciplinary collaboration makes it possible to create a much-needed and commercial solution. 


\section{REFERENCES}

Adams, R., Wilson, M., and Wang, W.-C. (1997). The multidimensional random coefficients multinomial logit model. Appl. Psychol. Meas. 21, 1-23. doi: $10.1177 / 0146621697211001$

Attali, Y. (2014). A ranking method for evaluating constructed responses. Educ. Psychol. Meas. 74, 795-808. doi: 10.1177/0013164414527450

Bradley, R. A., and Terry, M. E. (1952). Rank Analysis Of Incomplete Block Designs: I. The method of paired comparisons. Biometrika 39:324. doi: 10.1093/biomet/39.3-4.324

Braithwaite, D. W., Goldstone, R. L., van der Maas, H. L. J., and Landy, D. H. (2016). Non-formal mechanisms in mathematical cognitive development: the case of arithmetic. Cognition 149, 40-55. doi: 10.1016/j.cognition.2016. 01.004

Brinkhuis, M. J., Bakker, M., and Maris, G. (2015). Filtering data for detecting differential development. J. Educ. Measure. 52, 319-338. doi: $10.1111 /$ jedm. 12078

Chen, Y., Li, X., Liu, J., and Ying, Z. (2018). Recommendation system for adaptive learning. Appl. Psychol. Meas. 42, 24-41. doi: 10.1177/0146621617697959

Coomans, F., Hofman, A., Brinkhuis, M., van der Maas, H. L., and Maris, G. (2016). Distinguishing fast and slow processes in accuracy-response time data. PLoS ONE 11:e0155149. doi: 10.1371/journal.pone.0155149

Corbett, A. T., and Anderson, J. R. (1994). Knowledge tracing: modeling the acquisition of procedural knowledge. User Model. UserAdap. Inter. 4, 253-278. doi: 10.1007/BF01099821

De Boeck, P., Chen, H., and Davison, M. (2017). Spontaneous and imposed speed of cognitive test responses, Br. J. Math. Stat. Psychol. 70, 225-237. doi: 10.1111/bmsp.12094

Doebler, P., Alavash, M., and Giessing, C. (2015). Adaptive experiments with a multivariate Elo-type algorithm. Behav. Res. Methods 47, 384-394. doi: 10.3758/s13428-014-0478-7

Eggen, T. J. H. M., and Verschoor, A. J. (2006). Optimal testing with easy or difficult items in computerized adaptive testing. Appl. Psychol. Meas. 30, 379-393. doi: $10.1177 / 0146621606288890$

Elo, A. E. (1978). The Rating of Chess Players, Past and Present. London: B.T. Batsford, Ltd.

Ferrini-Mundy, J., and Schmidt, W. H. (2005). International comparative studies in mathematics education: opportunities for collaboration and challenges for researchers. J. Res. Math. Educ. 36, 164-175. doi: 10.1007/978-3-319-42414-9_1

Gelman, A., and Rubin, D. B. (1995). Avoiding model selection in Bayesian social research. Discussion of "Bayesian model selection in social research." Soc. Method. 25, 165-173.

Glickman, M. E. (1999). Parameter estimation in large dynamic paired 1031 comparison experiments. Appl. Stat. 48, 377-394.

Hartig, J., and Höhler, J. (2008). Representation of competencies in multidimensional IRT models with within-item and between-item multidimensionality. J. Psychol. 216, 89-101. doi: 10.1027/0044-3409.216.2.89

Hofman, A., Jansen, B., de Mooij, S., Stevenson, C., and van der Maas, H. (2018). A solution to the measurement problem in the idiographic approach using computer adaptive practicing. J. Intel. 6:14. doi: 10.3390/jintelligence6010014

Kiraly, F. J., and Qian, Z. (2017). Modelling Competitive Sports: Bradley-Terry-Elo Models for Supervised and Online Learning of Paired Competition Outcomes. arXiv 1701.08055.

Klinkenberg, S., Straatemeier, M., and van der Maas, H. L. J. (2011). Computer adaptive practice on Maths ability using a new item response model for on the fly ability and difficulty estimation. Comput. Educ. 57, 1813-1824. doi: 10.1016/j.compedu.2011.02.003

Linsen, S., Torbeyns, J., Verschaffel, L., Reynvoet, B., and De Smedt, B. (2016). The association between symbolic and nonsymbolic numerical magnitude processing and mental versus algorithmic subtraction in adults. Acta Psychol. 165, 34-42. doi: 10.1016/j.actpsy.2016.01.008

Maris, G., and Van der Maas, H. (2012). Speed-accuracy response models: scoring rules based on response time and accuracy. Psychometrika 77, 615-633. doi: 10.1007/s11336-012-9288-y
NiŽnan, J., Pálanek, R., and Rihák, J. (2015). "Student models for prior knowledge estimation," in Proceedings of the 8th International Conference on Educational Data Mining. (Madrid: International Educational Data Mining Society), 109-116.

Pálanek, R. (2016). Applications of the Elo rating system in adaptive educational systems. Comput. Educ. 98, 169-179. doi: 10.1016/j.compedu.2016.03.017

Papousek, J., Pelanek, R., and Stanislav, V. (2014). "Adaptive practice of facts in domains with varied prior knowledge," in Proceedings of the International Conference on Educational Data Mining (EDM), 6-13.

Park, J. Y., Joo, S. H., Cornillie, F., Van der Maas, H. L. J., and Van den Noortgate, W. (2018). An explanatory item response theory method for alleviating the cold-start problem in adaptive learning environments. Behav. Res. Method. doi: 10.3758/s13428-018-1166-9. [Epub ahead of print].

Plummer, M. (2015). Just Another Gibbs Sampler (JAGS). Retrieved from http:// mcmc--jags.sourceforge.net

R Core Team (2013). R: A Language and Environment for Statistical 1069 Computing (version 3.3.3). Vienna: R Foundation for 1070 Statistical Computing. Retrieved from: www.R-project.org

Reckase, M. D. (1985). The difficulty of test items that measure more than one ability. Appl. Psychol. Measure. 9, 401-441.

Robbins, H., and Monro, S. (1951). A Stochastic Approximation Method. Ann. Math. Stat. 22:400.

Savi, A. O., van der Maas, H. L., and Maris, G. K. (2015). Navigating massive open online courses. Science 347, 958-958. doi: 10.1126/science.347.6225.958

Su, Y. S., and Yajima, M. (2015). R2jags: Using R to Run 'JAGS'. R Package Version $0.5-7$.

Tang, X., Chen, Y., Li, X., Liu, J., and Ying, Z. (2018). A reinforcement learning approach to personalized learning recommendation systems. Br. J. Math. Stat. Psychol. 72, 108-135. doi: 10.1111/bmsp.12144

Tuerlinckx, F., and De Boeck, P. (2005). Two interpretations of the discrimination parameter. Psychometrika 70, 629-650. doi: 10.1007/s11336-000-0810-3

van der Linden, W. J. (2000). "Constrained adaptive testing with shadow tests," in, Computerized adaptive testing: Theory and practice. eds W. J. van der Linden and C. A. W. Glas (Boston, MA: Kluwer), 27-52. doi: 10.1007/0-306-475 31-6_2

van der Linden, W. J. (2007). A hierarchical framework for modeling speed and accuracy on test items. Psychometrika 72:287. doi: 10.1007/s11336-006-1 478-z

van der Linden, W. J. (2009). Conceptual issues in response-time modeling. J. Educ. Measure. 46, 247-272. doi: 10.1111/j.1745-3984.2009.0 0080.x

van Groen, M. M., Eggen, T. J. H. M., and Veldkamp, B. P. (2014). Item selection methods based on multiple objective approaches for classifying respondents into multiple levels. Appl. Psychol. Meas. 38, 187-200. doi: 10.1177/0146621613509723

Wauters, K., Desmet, P., and Van Den Noortgate, W. (2010). Adaptive item-based learning environments based on the item response theory: possibilities and challenges. J. Comput. Assist. Learn. 26, 549-562. doi: 10.1111/j.1365-2729.2010.00368.x

Wauters, K., Desmet, P., and Van Den Noortgate, W. (2012). Item difficulty estimation: an auspicious collaboration between data and judgment. Comput. Educ. 58, 1183-1193. doi: 10.1016/j.compedu.2011.11.020

Conflict of Interest Statement: The authors declare that the research was conducted in the absence of any commercial or financial relationships that could be construed as a potential conflict of interest.

Copyright (c) 2019 Park, Cornillie, van der Maas and Van Den Noortgate. This is an open-access article distributed under the terms of the Creative Commons Attribution License (CC BY). The use, distribution or reproduction in other forums is permitted, provided the original author(s) and the copyright owner(s) are credited and that the original publication in this journal is cited, in accordance with accepted academic practice. No use, distribution or reproduction is permitted which does not comply with these terms. 